\title{
Error Probability of DPSK Signals With Cross-Phase Modulation Induced Nonlinear Phase Noise
}

\author{
Keang-Po Ho, Senior Member, IEEE
}

\begin{abstract}
The error probability is derived analytically for differential phase-shift keying (DPSK) signals contaminated by both self- and cross-phase modulation (SPM and XPM)-induced nonlinear phase noise. XPM-induced nonlinear phase noise is modeled as Gaussian distributed phase noise. When fiber dispersion is compensated perfectly in each fiber span, XPM-induced nonlinear phase is summed coherently span after span and is the dominant nonlinear phase noise for typical wavelength-division-multiplexed (WDM) DPSK systems. For systems without or with XPM-suppressed dispersion compensation, SPM-induced nonlinear phase noise is usually the dominant nonlinear phase noise. With longer walkoff length, for the same mean nonlinear phase shift, 10-Gb/s systems are more sensitive to XPM-induced nonlinear phase noise than $40-\mathbf{G b} / \mathbf{s}$ systems.
\end{abstract}

Index Terms-Cross-phase modulation, differential phase-shift keying (DPSK), fiber nonlinearities, nonlinear phase noise, phase modulation.

\section{INTRODUCTION}

$\mathbf{N}$ ONLINEAR phase noise is induced by the interaction of fiber Kerr effect and optical amplifier noises when optical amplifiers are used periodically to compensate for fiber loss. Self-phase modulation (SPM)-induced nonlinear phase noise, often called the Gordon-Mollenauer effect [1], is the major degradation of single-channel differential phase-shift keying (DPSK) signals [1]-[9]. Recently, there has been renewed interest in using wavelength-division-multiplexed (WDM) DPSK systems for long-haul transmission systems [9]-[14] and WDM differential quadrature phase-shift keying (DQPSK) systems for spectrally efficient transmission [15]-[19]. While the impact of Gordon-Mollenauer effect on single-channel DPSK systems is well known [7], [8], [20], to our knowledge, other than the studies of [21] and [22] on phase noise variance or frequency response, the impact of XPM-induced nonlinear phase noise on the error probability of DPSK signals has not been studied.

Added directly to the signal phase, SPM-induced nonlinear phase noise is non-Gaussian-distributed [5], [23], [24] but XPM-induced nonlinear phase noise is Gaussian-distributed. Induced by many other adjacent WDM channels, the Gaussian convergence of XPM-induced nonlinear phase noise comes directly from the central limit theorem [25]. Independent of both SPM-induced nonlinear phase noise and the additive

Manuscript received October 3, 2003; revised January 16, 2004. This work was supported in part by the National Science Council of Taiwan under Grant NSC-92-2218-E-002-034.

The author is with the Institute of Communication Engineering and Department of Electrical Engineering, National Taiwan University, 106 Taipei, Taiwan, R.O.C. (e-mail: kpho@cc.ee.ntu.edu.tw).

Digital Object Identifier 10.1109/JSTQE.2004.826574 amplifier noises, XPM-induced nonlinear phase noise has the same mathematical model as laser phase noise for performance analysis [26].

When many additive phase noise components are assumed to be independent of each other, the Fourier coefficients of the overall phase noise are the product of the Fourier coefficients of each individual component. This paper combines the models of [8] and [26] after the variance of XPM-induced nonlinear phase noise is evaluated, mostly using the methods of [27] and [28].

This paper are organized as follows. Section II derives the nonlinear phase noise variance due to XPM for multispan WDM DPSK systems and compares it with that due to SPM. Section III calculates the error probability of a DPSK signal with both SPM- and XPM-induced nonlinear phase noise. Sections IV and V are the discussion and conclusion for this paper, respectively.

\section{Nonlinear Phase Noise VARIANCE Due tO CROSS-PHASE MODULATION}

This section derives the ratio of the variance of nonlinear phase noise induced by XPM to that by SPM. The variance is first derived for the two-channel pump-probe model and later extended to multichannel and multispan WDM systems.

\section{A. Pump-Probe Model}

Based on the pump-probe model of [21] and [27]-[29], the phase modulation of channel 1 (probe) induced by channel 2 (pump) is

$$
\phi_{1, \mathrm{XPM}}(L, t)=2 \gamma \int_{0}^{L} P_{2}\left(0, t+d_{12} z\right) e^{-\alpha z} \mathrm{~d} z
$$

where $P_{2}(z, t)$ is the power of channel 2 as a function of position $z$ and time $t, P_{2}(0, t)$ is the launched signal at the transmitter, $\gamma$ is the nonlinear coupling coefficient, $\alpha$ is the fiber attenuation coefficient, $L$ is the fiber length, $d_{12} \approx D \Delta \lambda$ is the relative walkoff between two channels with wavelength separation of $\Delta \lambda$, and $D$ is the dispersion coefficient.

By taking the Fourier transform of the autocorrelation function, when the power spectral density of $P_{2}(0, t)$ is $\Phi_{P_{2}}(f)$, the power spectral density of $\phi_{1, \mathrm{XPM}}(L, t)$ is

$$
\Phi_{\phi_{1}}(f)=\Phi_{P_{2}}(f)\left|H_{12}(f)\right|^{2}
$$

where $H_{12}(f)=2 \gamma \int_{0}^{L} e^{-\alpha z+j 2 \pi f d_{12} z} \mathrm{~d} z$ or [27], [28]

$$
H_{12}(f)=2 \gamma \frac{1-e^{-\alpha L+j 2 \pi f d_{12} L}}{\alpha-j 2 \pi f d_{12}} .
$$


At the DPSK receiver, after the asymmetric Mach-Zehnder interferometer [10], [11], [20], the differential nonlinear phase noise of $\Delta \phi_{1, \mathrm{XPM}}(L, t)=\phi_{1, \mathrm{XPM}}(L, t)-\phi_{1, \mathrm{XPM}}(L, t-$ $T$ ) adds to the differential phase of the signal, where $T$ is the symbol interval. Similar to [22], the power spectral density of $\Delta \phi_{1, \mathrm{XPM}}(L, t)$ is

$$
\Phi_{\Delta \phi_{1}}(f)=4 \Phi_{P_{2}}(f)\left|H_{12}(f)\right|^{2} \sin ^{2}(\pi f T) .
$$

When the pump (channel 2) has amplifier noises, $P_{2}(0, t)=$ $\left|E_{2}+N_{2}\right|^{2}$, where $E_{2}$ and $N_{2}$ are the electric fields for signal and noise, respectively. In the power of $P_{2}(0, t)=\left|E_{2}\right|^{2}+E_{2}$. $N_{2}^{*}+E_{2}^{*} \cdot N_{2}+\left|N_{2}\right|^{2}$, the dc term of $\left|E_{2}\right|^{2}$ gives no nonlinear phase noise but a constant phase shift, the signal-noise beating of $E_{2} \cdot N_{2}^{*}+E_{2}^{*} \cdot N_{2}$ gives a noise spectral density of $2\left|E_{2}\right|^{2} S_{\mathrm{sp}}$, and the noise-noise beating of $\left|N_{2}\right|^{2}$ gives a noise spectral density of $2 S_{\mathrm{sp}}^{2} \Delta \nu_{\mathrm{opt}}$, where $S_{\mathrm{sp}}$ is the spectral density of the amplifier noise and $\Delta \nu_{\mathrm{opt}}$ is the optical bandwidth of the amplifier noise. The optical signal-to-noise ratio (SNR) over an optical bandwidth of $\Delta \nu_{\mathrm{opt}}$ is $\left|E_{2}\right|^{2} /\left(2 S_{\mathrm{sp}} \Delta \nu_{\mathrm{opt}}\right)$. For a launched power of $P_{0}$ and a single optical amplifier with a noise variance of $S_{\mathrm{sp}, 1}$, we get $\Phi_{P_{2}}(f)=n_{0}=2 P_{0} S_{\mathrm{sp}, 1}+2 S_{\mathrm{sp}, 1}^{2} \Delta \nu_{\mathrm{opt}}$. The phase variance as a function of frequency separation is

$$
\sigma_{\mathrm{XPM}, 0}^{2}(\Delta \lambda)=4 n_{0} \int_{-\frac{1}{T}}^{\frac{1}{T}}\left|H_{12}(f)\right|^{2} \sin ^{2}(\pi f T) \mathrm{d} f
$$

where the integration is reduced from $\pm \infty$ to $\pm 1 / T$ by taking into account only the phase noise over a bandwidth confined within the bit rate. The dependence of the variance of (5) on $\Delta \lambda$ is originated from the dependence of $H_{12}(f)$ of (3) on $\Delta \lambda$.

For SPM-induced nonlinear phase noise, using (5), we get $\sigma_{\mathrm{SPM}}^{2}=(1 / 4) \sigma_{\mathrm{XPM}, 0}^{2}(0)=4 n_{0} \gamma^{2} L_{\mathrm{eff}}^{2} / T$, where $L_{\mathrm{eff}}=(1-$ $\left.e^{-\alpha L}\right) / \alpha$ is the effective length per span. The factor of $1 / 4$ is because phase shift induced by XPM is twice as large as that induced by SPM for the same intensity. For a long fiber span with $L \gg 1 / \alpha$ and large walkoff coefficient of $d_{12}$, using [30, $\S 3.824]$, we get

$$
\begin{aligned}
\frac{\sigma_{\mathrm{XPM}, 0}^{2}(\Delta \lambda)}{\sigma_{\mathrm{SPM}}^{2}} & =8 \alpha^{2} T \int_{0}^{\frac{1}{T}} \frac{\sin ^{2}(\pi f T)}{\alpha^{2}+\left(2 \pi f d_{12}\right)^{2}} \mathrm{~d} f \\
& \approx 8 \alpha^{2} T \int_{0}^{\infty} \frac{\sin ^{2}(\pi f T)}{\alpha^{2}+\left(2 \pi f d_{12}\right)^{2}} \mathrm{~d} f \\
& =\alpha L_{W}\left(1-e^{-\alpha L_{W}}\right)
\end{aligned}
$$

where $L_{W}=T /\left|d_{12}\right|$ is the walkoff length that is the distance for two aligned pump and probe pulses of length $T$ becomes completely walkoff [31]. Like that for stimulated Raman scattering [31], even without approximation, the variances of XPMinduced nonlinear phase noise depend on the walkoff length of $L_{W}$ only.

\section{B. Multi-Span WDM Systems}

For a $(2 M+1)$-channel WDM system, for the worst case of the center channel, in the first span, the nonlinear phase noise variance per span is equal to

$$
\sigma_{\mathrm{XPM}, 1}^{2}=2 \sum_{k=1}^{M} \sigma_{\mathrm{XPM}, 0}^{2}(k \Delta \lambda)
$$

where, using the same symbol as the above pump-probe model, $\Delta \lambda$ is the wavelength separation between adjacent channels. For a short walkoff length of $L_{W}$ per channel separation of $\Delta \lambda$, a wavelength separation of $k \Delta \lambda$ gives a XPM-induced nonlinear phase noise variance of about a factor of $1 / k^{2}$ smaller than that for a wavelength separation of $\Delta \lambda$. For a large number of channels, using the relationship of $\sum_{k=1}^{\infty}\left(1 / k^{2}\right)=\left(\pi^{2} / 6\right)[30$, $\S 0.233$, we get

$$
\lim _{M \rightarrow \infty} \frac{\sigma_{\mathrm{XPM}, 1}^{2}}{\sigma_{\mathrm{SPM}}^{2}} \approx \frac{\pi^{2}}{3} \alpha L_{W}\left(1-e^{-\alpha L_{W}}\right) .
$$

For a system with $N$ fiber spans, the variance of the overall XPM-induced nonlinear phase noise depends on the method of dispersion compensation. Similar to (4), the power spectrum density of XPM-induced nonlinear phase noise after $K$ fiber spans is [28]

$$
\begin{aligned}
\Phi_{K, \Delta \phi_{1}}(f)= & 4 \Phi_{P_{2}}(f)\left|H_{12}(f)\right|^{2} \sin ^{2}(\pi f T) \\
& \times\left|\frac{\sin \left[K \pi f(1-\kappa) d_{12} L\right]}{\sin \left[\pi f(1-\kappa) d_{12} L\right]}\right|^{2}
\end{aligned}
$$

where $\kappa$ is the fraction of dispersion compensation, i.e., $\kappa=1$ and $\kappa=0$ for perfect and without dispersion compensation, respectively.

In the worst case of perfect dispersion compensation with $\kappa=$ 1 such that all channels are well aligned when launched to each fiber span, the variance of (5) is increased by a factor of $K^{2}$ after $K$ fiber spans. With perfect dispersion compensation, the variance of the overall XPM-induced nonlinear phase noise for an $N$-span fiber system is

$\sigma_{\mathrm{XPM}, \max }^{2}=N^{2} \sigma_{\mathrm{XPM}, 1}^{2}+(N-1)^{2} \sigma_{\mathrm{XPM}, 2}^{2}+\cdots+\sigma_{\mathrm{XPM}, N}^{2}$

where the first term of $\sigma_{\mathrm{XPM}, 1}^{2}$ is the nonlinear phase noise induced from the amplifier noise from the first span, the second term is the nonlinear phase noise induced from the amplifier noise from the second span, and so on. Because of perfect dispersion compensation, the same noise from the first span is perfectly (or coherently) added $N$ times into the overall nonlinear phase noise. The noise from the second $\operatorname{span} \sigma_{\mathrm{XPM}, 2}^{2}$ adds into the overall nonlinear phase noise $N-1$ times. With the assumption that all spans have the same configuration, $\sigma_{\mathrm{XPM}, 1}^{2}=$ $\sigma_{\mathrm{XPM}, 2}^{2}=\cdots=\sigma_{\mathrm{XPM}, N}^{2}$. The maximum nonlinear phase noise of (10) is

$$
\sigma_{\mathrm{XPM}, \max }^{2}=\frac{1}{6} N(N+1)(2 N+1) \sigma_{\mathrm{XPM}, 1}^{2} .
$$

For SPM-induced nonlinear phase noise, the amplifier noise from the first span is also perfectly aligned in all fiber spans afterward. The relationship between single- and multispan phase 
noise variance is the same as that of (11). With perfect dispersion compensation, the ratio of the variance of XPM- to SPM-induced nonlinear phase noise is independent of the number of fiber spans in WDM systems. The ratio of (8) can be used to approximate the ratio of XPM- to SPM-induced nonlinear phase noise in the worst case of perfect dispersion compensation.

When dispersion compensation is conducted channel-bychannel with XPM suppression [32], [33], the factor of $\kappa$ is the offset of pump and probe at the output of a fiber span with respect to the fiber walkoff. If $|\kappa| \gg 1$ such that the nonlinear phase noise induced to every fiber span is independent of each other, we get

$$
\sigma_{\mathrm{XPM}, \text { ind }}^{2}=N \sigma_{\mathrm{XPM}, 1}^{2}+(N-1) \sigma_{\mathrm{XPM}, 2}^{2}+\cdots+\sigma_{\mathrm{XPM}, N}^{2}
$$

or

$$
\sigma_{\mathrm{XPM}, \text { ind }}^{2}=\frac{1}{2} N(N+1) \sigma_{\mathrm{XPM}, 1}^{2}
$$

and the ratio of

$$
\frac{\sigma_{\mathrm{XPM}, \max }^{2}}{\sigma_{\mathrm{XPM}, \text { ind }}^{2}}=\frac{1}{3}(2 N+1) .
$$

In (12), the amplifier noise from the first span induces nonlinear phase noise in each fiber span. However, the nonlinear phase noises induced to the first and second span are from the amplifier noise in completely nonoverlapped time interval and independent of each other. In an $N$-span system, the overall nonlinear phase noise is generated from $(1 / 2) N(N+1)$ "pieces" of independent amplifier noise. The variance of (12) is also valid when $L \gg L_{W}$ and $\kappa \neq 1$.

The last term of (9) due to multispan effect of $\sin [K \pi f(1-$ $\left.\kappa) d_{12} L\right] / \sin \left[\pi f(1-\kappa) d_{12} L\right]$ has peak values of $K$ or $-K$ when $f(1-\kappa) d_{12} L=k$, where $k$ is a positive or negative integer. The last term of (4) due to differential operation of $\sin ^{2}(\pi f T)$ has notches at $f=k / T$. If the notches of $\sin ^{2}(\pi f T)$ match to the peaks of $\sin \left[K \pi f(1-\kappa) d_{12} L\right] / \sin \left[\pi f(1-\kappa) d_{12} L\right]$, XPMinduced nonlinear phase noise is approximately minimized. If $L_{W}<L$, the dispersion compensation factor of

$$
\kappa=1-\frac{L_{W}}{L}
$$

approximately gives minimum XPM-induced nonlinear phase noise. With resonance effect [34], certain combinations of walkoff length and dispersion compensation factor minimize the variance of XPM-induced nonlinear phase noise. Numerical results show that the compensation factor of (15) approximately minimizes the variance of XPM-induced nonlinear phase noise.

Fig. 1 shows the ratio of the standard deviation of XPMto SPM-induced nonlinear phase noise as a function of the walkoff length $L_{W}$ per channel separation $\Delta \lambda$. The ratio of $\sigma_{\mathrm{XPM}, \min } / \sigma_{\mathrm{SPM}}$ is calculated numerically and uses the dispersion compensation factor of (15) for $L_{W}<L$ and $\kappa=0$ for $L_{W} \geq L$. The fiber attenuation coefficient is $\alpha=0.21 \mathrm{~dB} / \mathrm{km}$. The fiber span length is $L=80 \mathrm{~km}$. For the pump-probe model (labeled as "2" WDM channels), the approximation of (6) is valid for $L_{W}$ less than $50 \mathrm{~km}$.

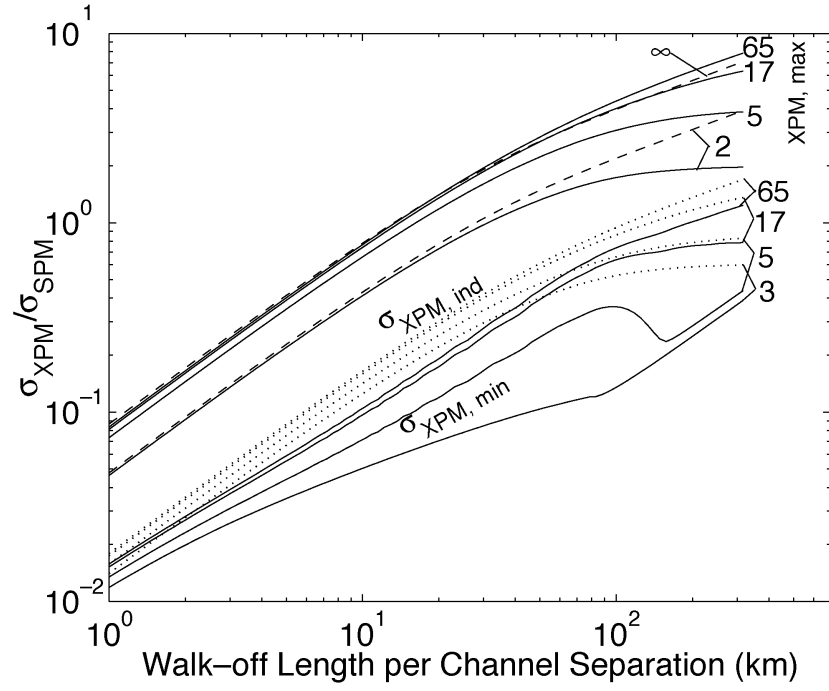

Fig. 1. The ratio of $\sigma_{\mathrm{XPM}} / \sigma_{\mathrm{SPM}}$ as a function of walkoff length per channel separation. The dashed curves are the approximation of (6) and (8). The ratio for the worst case of $\sigma_{\mathrm{XPM} \text {, max }}$ is independent of the number of spans. The ratios for the typical cases of $\sigma_{\mathrm{XPM} \text {,ind }}$ and the minimum case of $\sigma_{\mathrm{XPM} \text {, min }}$ are for $N=32$ fiber spans and are shown as dotted and solid lines, respectively. The label of each curve is the number of WDM channels.

For multichannel WDM systems, in both the worst and independent cases, the approximation of (8) is valid for $L_{W}$ less than $100 \mathrm{~km}$ and a number of channels larger than 17 . The approximation of (8) can be used to model typical WDM systems. For example, a typical $10-\mathrm{Gb} / \mathrm{s} 50-\mathrm{GHz}$ spacing system using nonzero dispersion-shifted fiber (NZDSF) with $D=4 \mathrm{ps} / \mathrm{km} / \mathrm{nm}$ has a walkoff length of $62.5 \mathrm{~km}$. Typical $40-\mathrm{Gb} / \mathrm{s}$ systems have a walkoff length of $7.8 \mathrm{~km}(100-\mathrm{GHz}$ spacing and $D=4 \mathrm{ps} / \mathrm{km} / \mathrm{nm}$ ).

When the walkoff effect is weak with long walkoff length $\left(L_{W}>100 \mathrm{~km}\right.$ ), from the pump-probe model, the nonlinear phase noise standard deviation due to XPM approaches twice that due to SPM. With long walkoff length, nonlinear phase noise induced by XPM is much larger than that induced by SPM.

For a multispan system, the variance of the SPM-induced nonlinear phase noise is equal to [1]

$$
\sigma_{\mathrm{SPM}}^{2} \approx \frac{4\left\langle\Phi_{\mathrm{NL}}\right\rangle^{2}}{3 \rho_{s}}
$$

with the exact results given in [35], where $\rho_{s}$ is the SNR defined for optical matched filter and a single polarization [7], [8]. The variance of (16) is twice that of [1], [35] for differential signal. Combining (16) with the ratio in Fig. 1, the variance of XPMinduced nonlinear phase noise $\sigma_{\mathrm{XPM}}^{2}$ can be calculated.

\section{ERROR PROBABILITY}

From [8], with only SPM-induced nonlinear phase noise, the error probability of a DPSK signal is

$$
\begin{gathered}
p_{e, \mathrm{SPM}}=\frac{1}{2}-\frac{\rho_{s} e^{-\rho_{s}}}{2} \sum_{k=0}^{\infty} \frac{(-1)^{k}}{2 k+1}\left[I_{k}\left(\frac{\rho_{s}}{2}\right)+I_{k+1}\left(\frac{\rho_{s}}{2}\right)\right]^{2} \\
\times\left|\Psi_{\Phi_{\mathrm{NL}}}(2 k+1)\right|^{2}
\end{gathered}
$$

where $k$ is a summation index, $I_{k}(\cdot)$ is a $k$ th-order modified Bessel function of the first kind, and $\Psi_{\Phi_{\mathrm{NL}}}(\nu)$ is the character- 


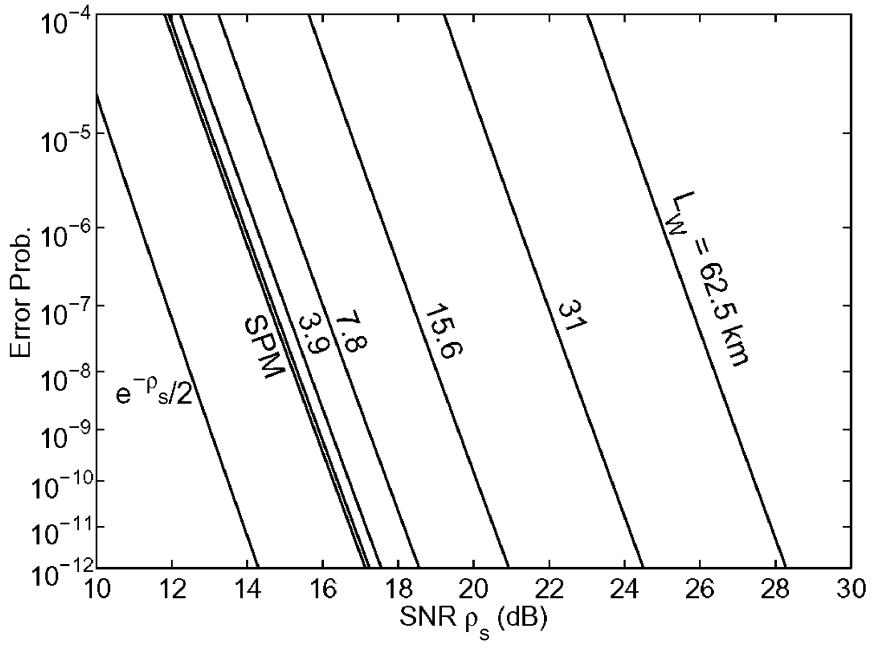

(a) $\sigma_{\mathrm{XPM}, \max }^{2}$

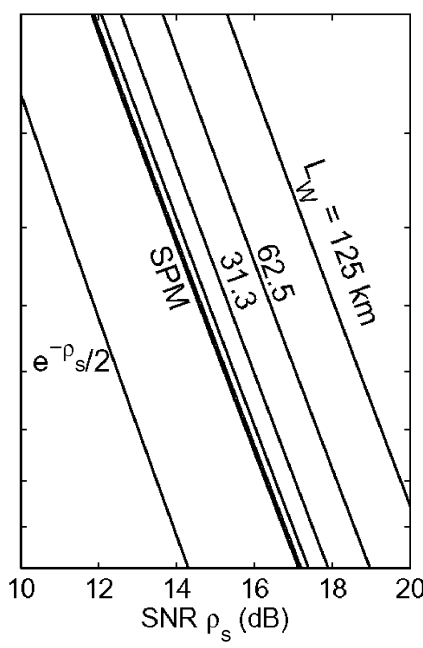

(b) $\sigma_{\mathrm{XPM}, \text { ind }}^{2}$

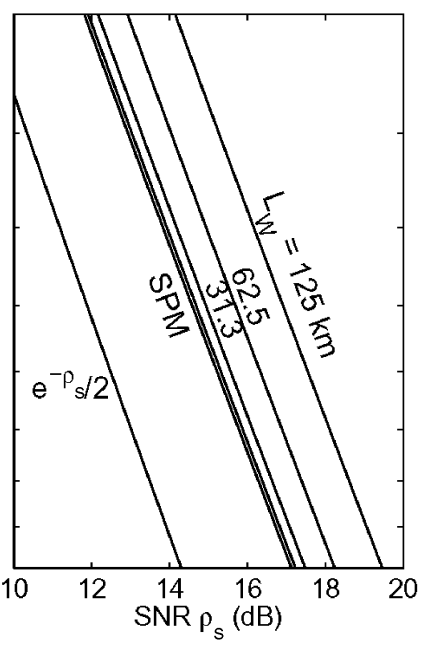

(c) $\sigma_{\mathrm{XPM}, \min }^{2}$

Fig. 2. Error probability as a function of SNR for WDM DPSK systems with various dispersion compensation schemes, corresponding to (a) maximum, (b) independent, and (c) minimum variance of XPM-induced nonlinear phase noise.

istic function of SPM-induced nonlinear phase noise given by [23]. Simulation by error counting confirms the validity of the error probability of (17) [8].

When independent phase noises from different sources are summed together, the coefficients of the Fourier series of the overall probability density function are the product of the corresponding Fourier coefficients of each individual component. If the XPM-induced nonlinear phase noise is Gaussian distributed, the error probability of the DPSK signal is

$$
\begin{aligned}
p_{e, \mathrm{XPM}}= & \frac{1}{2}-\frac{\rho_{s} e^{-\rho_{s}}}{2} \sum_{k=0}^{\infty} \frac{(-1)^{k}}{2 k+1}\left[I_{k}\left(\frac{\rho_{s}}{2}\right)+I_{k+1}\left(\frac{\rho_{s}}{2}\right)\right]^{2} \\
& \times\left|\Psi_{\Phi_{\mathrm{NL}}}[(2 k+1) j]\right|^{2} \exp \left[-\frac{2 k+1}{2} \sigma_{\mathrm{XPM}}^{2}\right] .(18)
\end{aligned}
$$

The formulas of (17) and (18) are similar to that with noisy reference [36], laser phase noise [26], phase error [37], or laser phase noise together with phase error [38]. The terms within the summation of (18) are the product of that due to SPM-induced nonlinear phase noise [8] and laser phase noise [26].

Because XPM-induced nonlinear phase noise is generated by the interaction of many bits or WDM channels, the Gaussian approximation is valid from the central limit theorem [25]. If the walkoff length of $L_{W}$ is small, the nonlinear phase noise is induced by at least about $2 \times L_{\text {eff }} / L_{W}$ independent bits from two adjacent channels [31], [39]. If the walkoff length is large, many adjacent channels induce more or less the same amount of nonlinear phase noise [31], [39], [40]. In both cases, the central limit theorem leads to Gaussian distribution.

Fig. 2 shows the error probability of DPSK signal as a function of SNR $\rho_{s}$. The error probability is calculated by (17) and (18) for a system with and without XPM-induced nonlinear phase noise, respectively. The system in Fig. 2 has $N=32$ identical fiber spans, mean nonlinear phase shift of $\left\langle\Phi_{\mathrm{NL}}\right\rangle=1 \mathrm{rad}$, and 65 WDM channels. The mean nonlinear phase shift of $1 \mathrm{rad}$ is the same as the estimation from [1] and close to the optimal operating point from [8]. Taking into account only SPM-induced nonlinear phase shift, the mean nonlinear phase shift can be calculated approximately from [1] and accurately from [35]. Fig. 2 also plots the error probability of $(1 / 2) e^{-\rho_{s}}$ without nonlinear phase noise [7], [8], [36], [37].

The walkoff length of Fig. 2 forms a geometric series and corresponds to typical 10- and 40-Gb/s systems in NZDSF and standard single-mode fiber (SSMF) with dispersion coefficients of $D=4$ and $16 \mathrm{ps} / \mathrm{km} / \mathrm{nm}$, respectively. For example, the walkoff length $L_{W}=7.8 \mathrm{~km}$ is that of a $10-\mathrm{Gb} / \mathrm{s} 100-\mathrm{GHz}$ spacing $(\Delta \lambda=0.8 \mathrm{~nm})$ system in SSMF and $40-\mathrm{Gb} / \mathrm{s} 100-\mathrm{GHz}$ spacing system in NZDSF.

Fig. 2(a)-(c) is calculated using the XPM-induced nonlinear phase noise variances of $\sigma_{\mathrm{XPM}, \max }^{2}, \sigma_{\mathrm{XPM} \text {,ind }}^{2}$, and $\sigma_{\mathrm{XPM} \text {,min }}^{2}$ in (18), respectively, corresponding to the worst, independent, and best case dispersion compensation, respectively. Comparing Fig. 2(b) and (c) with Fig. 2(a), the DPSK system requires the reduction of XPM-induced nonlinear phase noise using, for example, XPM suppressor [32], [33] or optimal dispersion compensation factor of (15). With perfect dispersion compensation, XPM-induced cross-phase modulation is negligible if $L_{W}<$ $3.9 \mathrm{~km}$. Without dispersion compensation or with XPM suppressor, XPM-induced cross-phase modulation is negligible if $L_{W}<15.8 \mathrm{~km}$.

Fig. 3 shows the SNR penalty for an error probability of $10^{-9}$ as a function of mean nonlinear phase shift of $\left\langle\Phi_{\mathrm{NL}}\right\rangle$ for the same system as Fig. 2. Fig. 3(a)-(c) is calculated using the XPM-induced nonlinear phase noise variances of $\sigma_{\mathrm{XPM} \text {, max }}^{2}$, $\sigma_{\mathrm{XPM}, \text { ind }}^{2}$, and $\sigma_{\mathrm{XPM}, \mathrm{min}}^{2}$ in (18), respectively, corresponding to the worst, independent, and best case dispersion compensation, respectively.

For the systems in Fig. 3(a), with perfect dispersion compensation such that XPM-induced nonlinear phase noise adds coherently, XPM-induced nonlinear phase noise gives the same SNR penalty as SPM-induced nonlinear phase noise when the walkoff length is about $L_{W}=7.8 \mathrm{~km}$ for $\left\langle\Phi_{\mathrm{NL}}\right\rangle$ less than $1 \mathrm{rad}$. For a $10-\mathrm{Gb} / \mathrm{s}$ WDM system in SSMF, the channel spacing must be 


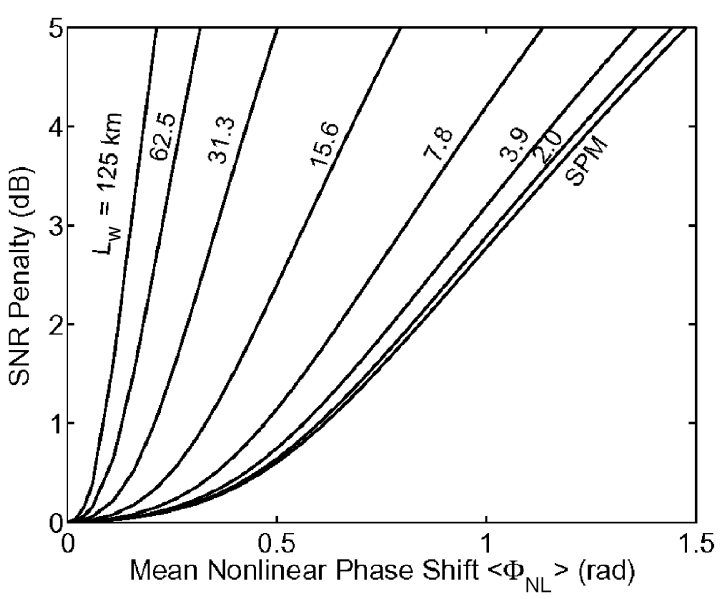

(a) $\sigma_{\mathrm{XPM}, \max }^{2}$

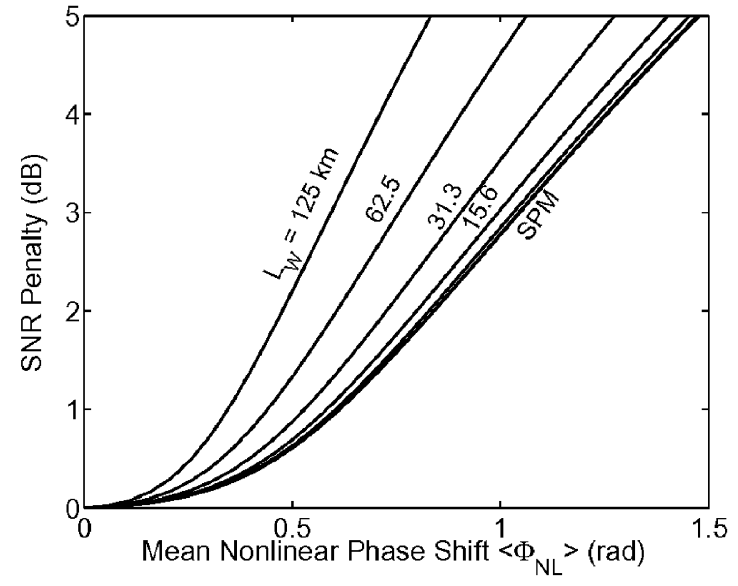

(b) $\sigma_{\mathrm{XPM} \text {,ind }}^{2}$

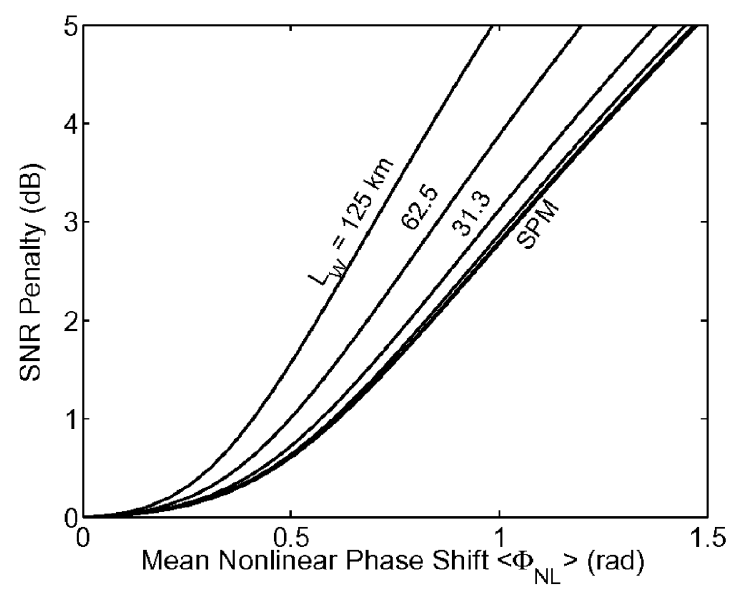

(c) $\sigma_{\mathrm{XPM}, \min }^{2}$

Fig. 3. SNR penalty as a function of mean nonlinear phase shift $\left\langle\Phi_{N L}\right\rangle$ for WDM DPSK systems with various dispersion compensation schemes, corresponding to (a) maximum, (b) independent, and (c) minimum variance of XPM-induced nonlinear phase noise.

larger than or equal to $100 \mathrm{GHz}$ (or $0.8 \mathrm{~nm}$ ). For a $10-\mathrm{Gb} / \mathrm{s}$ WDM system in NZDSF, the channel spacing must be larger than or equal to $400 \mathrm{GHz}$ (or $3.2 \mathrm{~nm}$ ). For a 10-Gb/s WDM system with typical channel spacing of 50 or $100 \mathrm{GHz}$, with perfect dispersion compensation, the effect of XPM-induced nonlinear phase noise is larger than that induced by SPM. For a 40-Gb/s WDM system in NZDSF, the channel spacing must be larger than or equal to $100 \mathrm{GHz}$. With channel spacing larger than $100 \mathrm{GHz}$, typical 40-Gb/s WDM systems have an SNR penalty from XPM-induced nonlinear phase noise about that from SPM.

For the systems in Fig. 3(b) and (c), with dispersion compensation factor of (15) or with XPM suppressor, XPM-induced nonlinear phase noise gives the same SNR penalty as SPM-induced nonlinear phase noise when the walkoff length is about $L_{W}=62.5 \mathrm{~km}$. For a $10-\mathrm{Gb} / \mathrm{s}$ WDM system in SSMF, the channel spacing must be larger than or equal to $12.5 \mathrm{GHz}$ (or $0.1 \mathrm{~nm}$ ). For a $10-\mathrm{Gb} / \mathrm{s}$ WDM system in NZDSF, the channel spacing must be larger than or equal to $50 \mathrm{GHz}$ (or $0.4 \mathrm{~nm}$ ). For a $10-\mathrm{Gb} / \mathrm{s}$ WDM system with typical channel spacing of 50 or $100 \mathrm{GHz}$, the effect of XPM-induced nonlinear phase noise is much smaller than that induced by SPM. Typical $40-\mathrm{Gb} / \mathrm{s}$ WDM systems have an SNR penalty from XPM-induced nonlinear phase noise far less than that from SPM.

\section{DISCUSSION}

In the derivation of (5), the term of $\left|E_{2}\right|^{2}$ is ignored. For a nonreturn-to-zero (NRZ) DPSK signal, $\left|E_{2}\right|^{2}$ is a dc term and can be ignored. For the more popular return-to-zero (RZ) DPSK signal [10], [13], $\left|E_{2}\right|^{2}$ is a periodic function with a period of $T$ and its power spectral density is tones at $k / T$, where $k$ is an integer. The differential transfer function of $2 \sin ^{2}(\pi f T)$ has notches at $k / T$ and cancels all nonlinear phase noise due to $\left|E_{2}\right|^{2}$ even for an RZ signal. For an RZ-DPSK signal with pulse broadening due to fiber dispersion, if the dipersion is assumed to be a linear effect, for a system without pulse overlapping, the differential transfer function can also completely eliminate XPM-induced nonlinear phase noise. Due to interferometer phase error [41]-[43], small residual nonlinear phase noise may be induced by those tones. The numerical results of this paper are also valid for flattop RZ-DPSK signals by increasing the mean nonlinear phase shift by a factor equal to the inverse of the RZ pulse duty cycle.

For the same channel spacing and fiber type, $10-\mathrm{Gb} / \mathrm{s}$ systems have a walkoff length four times that of $40-\mathrm{Gb} / \mathrm{s}$ systems. From Fig. 3, with the same mean nonlinear phase shift, the nonlinear phase-noise-induced SNR penalty of $10-\mathrm{Gb} / \mathrm{s}$ systems is smaller than that for the corresponding $40-\mathrm{Gb} / \mathrm{s}$ systems. However, $40-\mathrm{Gb} / \mathrm{s}$ systems have four times the bandwidth and re- 
quire four times the power of the corresponding $10-\mathrm{Gb} / \mathrm{s}$ systems having the same SNR $\rho_{s}$ defined in [7] and [8]. Because the mean nonlinear phase shift is proportional to the launched power, for the same SNR and system configuration, the mean nonlinear phase shift of $40-\mathrm{Gb} / \mathrm{s}$ systems is four times larger than that for $10-\mathrm{Gb} / \mathrm{s}$ systems.

When the dependence between nonlinear phase noise and amplifier noise is taken into account, we derive the exact error probability of DPSK signals with nonlinear phase noise when the number of fiber spans approaches infinity [20]. With the distributed assumption, the difference between exact and approximate error probability is less than $0.23 \mathrm{~dB}$ in terms of SNR penalty. Currently, the exact error probability of DPSK signals with finite number of fiber spans is not known. From the simulation of [8], the model of (17) is very accurate.

SPM-induced nonlinear phase noise correlates with the received intensity and can be compensated using the correlation properties [8], [20], [35], [44], [45]. Other than using simultaneous multichannel detection, XPM-induced nonlinear phase noise cannot be compensated.

Due to fiber dispersion, phase modulation (PM) converts to amplitude-modulation (AM) noise. Combined with XPM, AM noise gives nonlinear phase noise to other channels [46]. When phase modulation converts to amplitude modulation, only high-frequency AM noise is induced by a transfer function of $\sin \left(\alpha f^{2}\right)$, where $\alpha$ is a constant depending on fiber length and dispersion [47]. With the low-pass characteristic of $H_{12}(f)$ of (3), the combined effects of AM-PM conversion with XPM should be small.

\section{CONClusion}

Closed-form formulas are derived for the error probability of WDM DPSK signals contaminated by both SPM- and XPM-induced nonlinear phase noise. The error probability is derived based on the assumption that the phase of amplifier noise is independent of nonlinear phase noise. While SPM-induced nonlinear phase noise is not Gaussian-distributed, XPM-induced nonlinear phase noise is assumed to be Gaussian distributed when either the walkoff length is small or the number of WDM channels is large.

When fiber dispersion is compensated perfectly in each fiber span, XPM-induced nonlinear phase is summed coherently span after span and is the dominant nonlinear phase noise for typical multispan WDM DPSK systems. For a system without dispersion compensation or with XPM suppressor, the dominant nonlinear phase noise is typically induced by SPM. In general, with longer walkoff length, 10-Gb/s systems are more likely to be dominated by XPM-induced nonlinear phase noise than $40-\mathrm{Gb} / \mathrm{s}$ systems.

\section{REFERENCES}

[1] J. P. Gordon and L. F. Mollenauer, "Phase noise in photonic communications systems using linear amplifiers," Opt. Lett., vol. 15, no. 23, pp. 1351-1353, 1990.

[2] S. Ryu, "Signal linewidth broadening due to nonlinear Kerr effect in long-haul coherent systems using cascaded optical amplifiers," J. Lightwave Technol., vol. 10, no. 10, pp. 1450-1457, 1992.

[3] S. Saito, M. Aiki, and T. Ito, "System performance of coherent transmission over cascaded in-line fiber amplifiers," J. Lightwave Technol., vol. 11 , no. 2, pp. 331-342, 1993
[4] A. Mecozzi, "Limits to long-haul coherent transmission set by the Kerr nonlinearity and noise of the in-line amplifiers," J. Lightwave Technol., vol. 12, no. 11, pp. 1993-2000, 1994.

[5] H. Kim and A. H. Gnauck, "Experimental investigation of the performance limitation of DPSK systems due to nonlinear phase noise," IEEE Photon. Technol. Lett., vol. 15, no. 2, pp. 320-322, 2003.

[6] C. Xu, X. Liu, L. F. Mollenauer, and X. Wei, "Comparison of return-to-zero differential phase-shift keying and on-off keying long-haul dispersion managed transmission," IEEE Photon. Technol. Lett., vol. 15, no. 4, pp. 617-619, 2003.

[7] K.-P. Ho, "Performance degradation of phase-modulated systems with nonlinear phase noise," IEEE Photon. Technol. Lett., vol. 15, no. 9, pp. 1213-1215, 2003.

[8] — , "Compensation improvement of DPSK signal with nonlinear phase noise," IEEE Photon. Technol. Lett., vol. 15, no. 9, pp. $1216-1218,2003$.

[9] T. Mizuochi, K. Ishida, T. Kobayashi, J. Abe, K. Kinjo, K. Motoshima, and K. Kasahara, "A comparative study of DPSK and OOK WDM transmission over transoceanic distances and their performance degradations due to nonlinear phase noise," J. Lightwave Technol., vol. 21, no. 9, pp. 1933-1943, 2003.

[10] A. H. Gnauck, G. Raybon, S. Chandrasekhar, J. Leuthold, C. Doerr, L. Stulz, A. Agrawal, S. Banerjee, D. Grosz, S. Hunsche, A. Kung, A. Marhelyuk, D. Maymar, M. Movassaghi, X. Liu, C. Xu, X. Wei, and D. M. Gill, " $2.5 \mathrm{~Tb} / \mathrm{s}(64 \times 42.7 \mathrm{~Gb} / \mathrm{s})$ transmission over 40 $\times 100 \mathrm{~km}$ NZDSF using RZ-DPSK format and all-Raman-amplified spans," in Optical Fiber Commun. Conf. (OFC '02), postdeadline paper FC2.

[11] A. H. Gnauck, G. Raybon, S. Chandrasekhar, J. Leuthold, C. Doerr, L. Stulz, and E. Burrows, " 25 40-Gb/s copolarized DPSK transmission over 12 100-km NZDF with 50-GHz channel spacing," IEEE Photon. Technol. Lett., vol. 15, no. 3, pp. 467-469, 2003.

[12] C. Rasmussen, T. Fjelde, J. Bennike, F. Liu, S. Dey, B. Mikkelsen, P. Mamyshev, P. Serbe, P. van de Wagt, Y. Akasaka, D. Harris, D. Gapontsev, V. Ivshin, and P. Reeves-Hall, "DWDM 40G transmission over trans-Pacific distance $(10,000 \mathrm{~km})$ using CSRZ-DPSK, enhanced FEC and all-Raman amplified $100 \mathrm{~km}$ Ultra-Wave fiber spans," in Optical Fiber Commun. Conf. (OFC '03), postdeadline paper PD18.

[13] B. Zhu, L. E. Nelson, S. Stulz, A. H. Gnauck, C. Doerr, J. Leuthold, L. Grüner-Nielsen, M. O. Pederson, J. Kim, R. Lingle, Y. Emori, Y. Ohki, N. Tsukiji, A. Oguri, and S. Namiki, "6.4-Tb/s $(160 \times 42.7 \mathrm{~Gb} / \mathrm{s})$ transmission with $0.8 \mathrm{bit} / \mathrm{s} / \mathrm{Hz}$ spectral efficiency over $32 \times 100 \mathrm{~km}$ of fiber using CSRZ-DPSK format," in Optical Fiber Commun. Conf. (OFC '03), postdeadline paper PD19.

[14] J.-X. Cai, D. G. Foursa, C. R. Davidson, Y. Cai, G. Domagala, H. Li, L. Liu, W. W. Patterson, A. N. Pilipetskii, M. Nissov, and N. S. Bergano, "A DWDM demonstration of $3.73 \mathrm{~Tb} / \mathrm{s}$ over $11000 \mathrm{~km}$ using 373 RZ-DPSK channels at $10 \mathrm{~Gb} / \mathrm{s}$," in Optical Fiber Commun. Conf. (OFC '03), postdeadline paper PD22.

[15] R. A. Griffin, R. I. Johnstone, R. G. Walker, J. Hall, S. D. Wadsworth, K. Berry, A. C. Carter, M. J. Wale, P. A. Jerram, and N. J. Parsons, " 10 $\mathrm{Gb} / \mathrm{s}$ optical differential quadrature phase shift key (DQPSK) transmission using GaAs/AlGaAs integration," in Optical Fiber Commun. Conf. (OFC'02), postdeadline paper FD6.

[16] R. A. Griffin and A. C. Carter, "Optical differential quadrature phaseshift key (oDQPSK) for high capacity optical transmission," in Optical Fiber Commun. Conf. (OFC '02), paper WX6.

[17] P. S. Cho, V. S. Grigoryan, Y. A. Godin, A. Salamon, and Y. Achiam, "Transmission of 25-Gb/s RZ-DQPSK signals with $25-\mathrm{GHz}$ channel spacing over $1000 \mathrm{~km}$ of SMF-28 fiber," IEEE Photon. Technol. Lett., vol. 15, no. 3, pp. 473-475, 2003.

[18] H. Kim and R.-J. Essiambre, "Transmission of $8 \times 20 \mathrm{~Gb} / \mathrm{s}$ DQPSK signals over $310-\mathrm{km}$ SMF with $0.8 \mathrm{~b} / \mathrm{s} / \mathrm{Hz}$ spectral efficiency," IEEE Photon. Technol. Lett., vol. 15, no. 5, pp. 769-771, 2003.

[19] C. Wree, N. Hecker-Denschlag, E. Gottwald, P. Krummrich, J. Leibrich, E.-D. Schmidt, and B. L. W. Rosenkranz, "High spectral efficiency $1.6-\mathrm{b} / \mathrm{s} / \mathrm{Hz}$ transmission $(8 \times 40 \mathrm{~Gb} / \mathrm{s}$ with a $25-\mathrm{GHz}$ grid) over $200-\mathrm{km}$ SSMF using RZ-DQPSK and polarization multiplexing," IEEE Photon. Technol. Lett., vol. 15, no. 9, pp. 1303-1305, 2003.

[20] K.-P. Ho, "Statistical properties of nonlinear phase noise," in Advances in Optics and Laser Research, W. T. Arkin, Ed. Hauppauge, NY: Nova Science, 2003, vol. 3.

[21] J. Leibrich, C. Wree, and W. Rosenkranz, "CF-RZ-DPSK for suppression of XPM on dispersion-managed long-haul optical WDM transmission on standard single-mode fiber," IEEE Photon. Technol. Lett., vol. 14, no. 2, pp. 215-217, 2002. 
[22] H. Kim, "Cross-phase-modulation-induced nonlinear phase noise in WDM direct-detection DPSK systems," J. Lightwave Technol., vol. 21, no. 8, pp. 1770-1774, 2003.

[23] K.-P. Ho, "Probability density of nonlinear phase noise," J. Opt. Soc. Amer. B, vol. 20, no. 9, pp. 1875-1879, 2003.

[24] _ "Asymptotic probability density of nonlinear phase noise," Opt. Lett., vol. 28, no. 15, pp. 1350-1352, 2003.

[25] A. Papoulis, Probability, Random Variables, and Stochastic Processes, 2nd ed. New York: McGraw-Hill, 1984.

[26] G. Nicholson, "Probability of error for optical heterodyne DPSK system with quantum phase noise," Electron. Lett., vol. 20, no. 24, pp. 1005-1007, 1984.

[27] T.-K. Chiang, N. Kagi, T. K. Fong, M. E. Marhic, and L. G. Kazovsky, "Cross-phase modulation in dispersive fibers: theoretical and experimental investigation of the impact of modulation frequency," IEEE Photon. Technol. Lett., vol. 6, no. 6, pp. 733-736, 1994.

[28] T.-K. Chiang, N. Kagi, M. E. Marhic, and L. G. Kazovsky, "Cross-phase modulation in fiber links with multiple optical amplifiers and dispersion compensators," J. Lightwave Technol., vol. 14, no. 3, pp. 249-260, 1996.

[29] K.-P. Ho, E. T.-P. Kong, L. Y. Chan, L.-K. Chan, and F. Tong, "Analysis and measurement of root-mean-squared bandwidth of cross-phase-modulation-induced spectral broadening," IEEE Photon. Technol. Lett., vol. 11, no. 9, pp. 1126-1128, 1999

[30] I. S. Gradshteyn and I. M. Ryzhik, Table of Integrals, Series, and Products. San Diego, CA: Academic, 1980.

[31] K.-P. Ho, "Statistical properties of simulated Raman crosstalk in WDM systems," J. Lightwave Technol., vol. 18, no. 7, pp. 915-921, 2000.

[32] G. Bellotti and S. Bigo, "Cross-phase modulation suppressor for multispan dispersion-managed WDM transmissions," IEEE Photon. Technol. Lett., vol. 12, no. 6, pp. 726-728, 2000.

[33] G. Bellotti, S. Bigo, P.-Y. Cortes, S. Gauchard, and S. LaRochelle, " $10 \times$ $10 \mathrm{~Gb} / \mathrm{s}$ cross-phase modulation suppressor for multispan transmissions using WDM narrow-band fiber Bragg gratings," IEEE Photon. Technol. Lett., vol. 12, no. 10, pp. 1403-1405, 2000.

[34] L. E. Nelson, R. M. Jopson, A. H. Gnauck, and A. R. Chraplyvy, "Resonances in cross-phase modulation impairment in wavelength-divisionmultiplexed lightwave transmission," IEEE Photon. Technol. Lett., vol. 11, no. 7, pp. 907-909, 1999.

[35] K.-P. Ho and J. M. Kahn, "Electronic compensation technique to mitigate nonlinear phase noise," J. Lightwave Technol., vol. 22, no. 3, pp. 779-783, 2004.

[36] P. C. Jain, "Error probabilities in binary angle modulation," IEEE Trans. Inform. Theory, vol. IT-20, no. 1, pp. 36-42, 1974.

[37] N. M. Blachman, "The effect of phase error on DPSK error probability," IEEE Trans. Commun., vol. COM-29, no. 3, pp. 364-465, 1981.

[38] G. Jacobsen and I. Garrett, "Theory for optical heterodyne DPSK receivers with post-detection filtering," J. Lightwave Technol., vol. LT-5, no. 4, pp. 478-484, 1987.

[39] F. Forghieri, R. W. Tkach, and A. R. Chraplyvy, "Fiber nonlinearities and their impact on transmission systems," in Optical Fiber Telecommunications, I. P. Kaminow and T. L. Koch, Eds. San Diego, CA: Academic, 1997, vol. IIIA, pp. 196-264.
[40] - "Effect of modulation statistics on Raman crosstalk in WDM systems," IEEE Photon. Technol. Lett., vol. 7, no. 1, pp. 101-103, 1995.

[41] H. Kim and P. J. Winzer, "Robustness to laser frequency offset in directdetection DPSK and DQPSK systems," J. Lightwave Technol., vol. 21, no. 9, pp. 1887-1891, 2003.

[42] P. J. Winzer and H. Kim, "Degradations in balanced DPSK receivers," IEEE Photon. Technol. Lett., vol. 15, no. 9, pp. 1282-1284, 2003.

[43] K.-P. Ho, "The effect of interferometer phase error on direct-detection DPSK and DQPSK signals," IEEE Photon. Technol. Lett., vol. 16, no. 1, pp. 308-310, 2004.

[44] X. Liu, X. Wei, R. E. Slusher, and C. J. McKinstrie, "Improving transmission performance in differential phase-shift-keyed systems by use of lumped nonlinear phase-shift compensation," Opt. Lett., vol. 27, no. 18, pp. 1616-1618, 2002

[45] C. Xu and X. Liu, "Postnonlinearity compensation with data-driven phase modulators in phase-shift keying transmission," Opt. Lett., vol. 27, no. 18, pp. 1619-1621, 2002.

[46] S. Norimatsu and K. Iwashita, "The influence of cross-phase modulation on optical FDM PSK homodyne transmission systems," J. Lightwave Technol., vol. 11, no. 5, pp. 795-804, 1993.

[47] J. Wang and K. Petermann, "Small signal analysis for dispersive optical fiber communication systems," J. Lightwave Technol., vol. 10, no. 1, pp. 96-100, 1992.

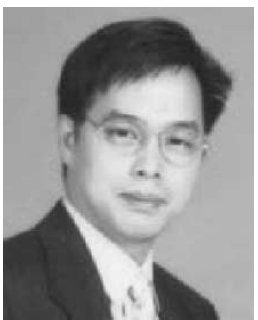

Keang-Po Ho (S'91-M'95-SM'03) received the B.S. degree from National Taiwan University, Taipei, Taiwan, R.O.C., in 1991 and the M.S. and Ph.D. degrees from the University of California at Berkeley in 1993 and 1995, respectively, all in electrical engineering.

He was with the IBM T. J. Watson Research Center, Hawthorne, NJ, in summer 1994, where he conducted research on all-optical networks. He was a Research Scientist with Bellcore (currently Telcordia Technologies), Red Bank, NJ, from 1995 to 1997, where he conducted research on optical networking, high-speed lightwave systems, and broadband access. He taught in the Department of Information Engineering, Chinese University of Hong Kong, from 1997 to 2001. He was Chief Technology Officer of StrataLight Communications, Campbell, CA, from 2000 to 2003, where he developed spectral efficiency 40-Gb/s lightwave transmission systems. He has been with the Institute of Communication Engineering and Department of Electrical Engineering, National Taiwan University, since 2003. His research interests include optical communication systems, multimedia communication systems, combined source-channel coding, and communication theory. He was among the pioneers for research on hybrid WDM systems, combined source-channel coding using multicarrier modulation or turbo codes, and performance evaluation of PSK and DPSK signals with nonlinear phase noise. He has published more than 130 journal and conference papers on those fields. 